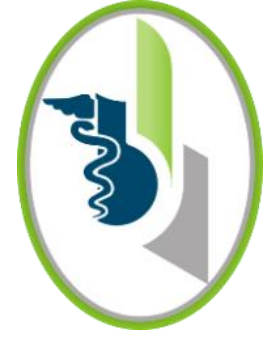

ACCESO $\odot$ ABIERTO

Para citaciones: Ricagni, L., Rivero, M. Aguiar, S., Sallabanda, K. (2021). Papel de la radiocirugía en el tratamiento de malformaciones arterio-venosas cerebrales. Revisión de la literatura y análisis de resultados. Revista Ciencias Biomédicas, 10(3), 189-197.

https://doi.org/10.32997/rcb-2021-3214

Recibido: 1 de febrero de 2021

Aprobado: 21 de abril de 2021

Autor de correspondencia:

Leandro Ricagni

leandroricagni@gmail.com

Editor: Inés Benedetti. Universidad de Cartagena-Colombia.

Copyright: (C) 2021. Ricagni, L., Rivero, M., Aguiar, S., Sallabanda, K.. Este es un artículo de acceso abierto, distribuido bajo los términos de la licencia

https://creativecommons.org/licenses/by-nc-

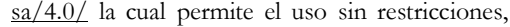
distribución y reproducción en cualquier medio, siempre y cuando el original, el autor y la fuente sean acreditados.

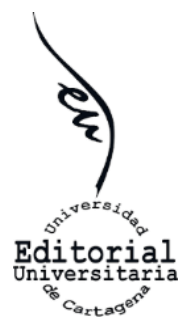

\section{Papel de la radiocirugía en el tratamiento de malformaciones arterio-venosas cerebrales. Revisión de la literatura y análisis de resultados}

\author{
Role of the radiosurgery in the treatment of cerebral arterio- \\ venous malformations. Review of the literature and analysis of \\ results
}

Leandro Ricagni $^{1}$ (D), Milexys Rivero Fuentes ${ }^{2}$ (D), Sergio Aguiar $^{3}$, Kita Sallabanda $^{4}$

${ }^{1}$ Departamento de Radioterapia, Hospital de clínicas “Dr. Manuel Quintela”, Montevideo, Uruguay.

${ }^{2}$ Especialización en Oncología radioterápica, Servicio de radioterapia, Hospital de clínicas "Dr. Manuel Quintela", Montevideo, Uruguay.

${ }^{3}$ Departamento de Radioterapia, Servicio de Oncología, Hospital de Clínicas "Dr. Manuel Quintela".

Uruguay.

${ }^{4}$ Programa de Master en Radiocirugía del Sistema Nerviosos Central, Universidad Complutense de Madrid, Madrid, España.

\section{RESUMEN}

Introducción: las malformaciones arteriovenosas (MAVs) son patologías infrecuentes, con una incidencia estimada de 1,1 en 100.000 habitantes. El tratamiento puede ser embolización vascular, microcirugía o radiocirugía estereotáctica. Por su baja incidencia, resulta difícil seleccionar un grupo homogéneo para un seguimiento prospectivo a largo plazo, y en base a resultados estadísticamente significativos definir una conducta.

Objetivo: establecer el rol de la radiocirugía estereotáctica en el tratamiento de malformaciones arteriovenosas cerebrales.

Métodos: se realizó una búsqueda en bases de datos para un análisis descriptivo de ensayos clínicos en pacientes con malformaciones arteriovenosas tratados con radiocirugía estereotáctica, sin radioterapia previa.

Resultados: fueron seleccionados 26 artículos. Los resultados provenientes en su mayoría de estudios retrospectivos. La obliteración estimada para malformaciones arteriovenosas pequeñas o moderadas con radiocirugía estereotáctica varía entre un $61 \%$ a $90 \%$, con una morbilidad menor a $5 \%$. La respuesta al tratamiento varía según el tamaño del nido, la dosis y la no embolización previa. Para las malformaciones arteriovenosas gigantes la tasa de obliteración descrita es del 35\% al 58\%, con una morbilidad que alcanza el $15 \%$.

Conclusión: la radiocirugía estereotáctica parece ser una opción efectiva y segura cuando no se plantea microcirugía, con tasas satisfactorias de obliteración, sin aumento del riesgo de sangrado en el periodo de latencia. En malformaciones arteriovenosas gigantes la radiocirugía muestra una menor tasa de respuesta completa por lo que el tratamiento intervencionista multimodal es justificable. La radiocirugía estereotáctica ha demostrado ser segura y efectiva, con un papel 
preponderante en malformaciones arteriovenosas pequeñas, profundas o en áreas elocuentes.

Palabras Clave: malformaciones arteriovenosas; radiocirugía; cirugía; embolización terapéutica; hemorragia.

\section{ABSTRACT}

Introduction: arteriovenous malformations are infrequent pathologies, with an estimated incidence of 1.1 in 100,000 inhabitants. Treatment can be vascular embolization, microsurgery, or stereotactic radiosurgery. Due to its low incidence, it is difficult to select a homogeneous group for long-term prospective follow-up, and based on statistically significant results to define a behavior.

Objective: to establish the role of stereotactic radiosurgery in the treatment of cerebral AVMs.

Methods: was performed a database research for a descriptive analysis of clinical trials in patients with arteriovenous malformations treated with stereotactic radiosurgery, without prior radiotherapy.

Results: 26 articles were selected. Most of the results come from retrospective studies. The estimated obliteration for small or moderate arteriovenous malformations with stereotactic radiosurgery ranges from $61 \%$ to $90 \%$, with a morbidity of less than $5 \%$. Response to treatment varies according to nest size, dose, and previous non-embolization. For giant arteriovenous malformations, the obliteration rate described is $35 \%$ to $58 \%$, with a morbidity that reaches $15 \%$.

Conclusions: stereotactic radiosurgery seems to be an effective and safe option when microsurgery is not considered, with satisfactory obliteration rates, without increased risk of bleeding in the latency period. In giant arteriovenous malformations, stereotactic radiosurgery shows a lower complete response rate, so multimodal interventional treatment is justifiable. Stereotactic radiosurgery has proven to be safe and effective, with a preponderant role in small, deep arteriovenous malformations or in eloquent areas.

Keywords: arteriovenous malformations; radiosurgery; surgery; therapeutic embolization; hemorrhage.

\section{INTRODUCCIÓN}

Las malformaciones arteriovenosas (MAVs) son patologías vasculares congénitas poco frecuentes. La incidencia de MAVs recién diagnosticadas varía de 1.12-1.34 por 100.000 personas-año, son más frecuentes en pacientes menores de 40 años, ambos sexos son igualmente afectados $y$ no se ha encontrado asociación con factores genéticos, demográficos o ambientales (1). La escala de
Spetzler-Martin (SM) es el sistema más comúnmente utilizado para la valoración quirúrgica de las MAVs, y su grado se deriva del tamaño del nido, la ubicación anatómica en el cerebro y el patrón de drenaje venoso de la MAV.

La tasa de hemorragia suele ser del 2 al $4 \%$ por año, con riesgo acumulado cuando no se tratan (2). La mortalidad asociada a la hemorragia varía de $12 \%$ a $66.7 \%$, y de los sobrevivientes un $23 \%$ a $40 \%$ tienen 
algún grado de incapacidad (3). Con una tasa de mortalidad general entre $0,7 \%$ a $2,9 \%$ por año (4).

En el estudio de Hermesniemi y colaboradores (5), pacientes con MAV no tratadas fueron seguidos desde el ingreso hasta la muerte, ruptura, o inicio del tratamiento. El riesgo promedio anual de hemorragia fue del 2,4\%. El riesgo fue mayor durante los primeros cinco años. Los factores de riesgo que predijeron una hemorragia subsiguiente fueron la edad temprana, la ruptura previa, las localizaciones profundas e infratentoriales y el drenaje venoso profundo. La ruptura previa, un gran tamaño y las ubicaciones infratentoriales y profundas fueron factores de riesgo independientes en los modelos multivariados.

Varios estudios han reportado un mayor riesgo de hemorragia en las MAVs que presentan aneurismas asociados, pero otros estudios lo contradicen $(6,7)$.
Entre las opciones intervencionistas están la embolización vascular, microcirugía o radioterapia. Cualquier manejo que se establezca se justifica solo cuando el riesgo de morbilidad y mortalidad de la intervención es menor que el riesgo del curso natural de la enfermedad.

La radiocirugía estereotáctica (SRS, como se conoce por sus siglas del inglés: Stereotactic Radio Surgery), es la técnica que se utiliza cuando se decide tratar con radioterapia. Consiste en la administración precisa de una alta dosis de radiación terapéuticamente segura y efectiva, a un blanco definido por imágenes (8). La obliteración de la MAV es el objetivo principal de la SRS, con lo que se busca prevenir la hemorragia. Para valorar el riesgo de tratamiento se aplica la clasificación de Pollok Flickinger que tiene en consideración el volumen de la MAV, la edad del paciente, la localización, si ha sido previamente embolizada o no, y el número de drenajes venosos (Tabla 1).

Tabla 1. Escala RBAS propuesta por Pollock y Flickinger

\section{Parámetros}

\begin{tabular}{ll}
\hline Volumen & $0.1 *$ Volumen $(\mathrm{ml} / \mathrm{cc})$ \\
\hline Edad & $0.2 *$ edad (años) \\
\hline Localización & $0.5 *$ localización \\
\hline Frontal/ Temporal & 0 \\
\hline Parietal /occipital /intraventraventricular /cuerpo calloso / cerebelo & 1 \\
\hline Tronco de encéfalo/ ganglios de la base/ Tálamo & 2 \\
\hline AVM score $=0.1 *$ volumen $+0.02 *$ edad $+0.5 *$ localización &
\end{tabular}

(8).

Los objetivos secundarios de la SRS son la preservación o mejora de la función neurológica, incluida la disminución de la epilepsia asociada a la MAV u otros signos o síntomas neurológicos asociados con el nido. La SRS actúa obliterando la MAV en un proceso que se da en dos etapas. Inicialmente la irradiación en altas dosis produce edema en vasos de pequeño y mediano tamaño, y vacuolización de las células endoteliales, posteriormente hay proliferación endotelial y depósitos de lípidos, hiperproliferación de células musculares lisas y depósito de material hialino, colágeno tipo IV y calcio en la matriz extracelular.
Esto lleva a un estrechamiento concéntrico de la luz del vaso. En una etapa posterior ocurre trombosis, fibrosis, y necrosis de los canales vasculares, que conducen a una respuesta duradera (9). Es importante destacar que este proceso puede tardar años (período de latencia), a diferencia de la microcirugía o la embolización que busca una obliteración inmediata.

\section{MÉTODOS}

Se realizó una búsqueda sistemática en PubMed y Cocrhane, de artículos publicados entre enero del 
2000 a agosto del 2020, en inglés o español, con las palabras "radiocirugía" AND "malformaciones arteriovenosas".

Con la lectura del abstract se seleccionaron aquellos ensayos clínicos que evaluaban el resultado de la SRS en MAVs rotas o no rotas, en pacientes mayores de 18 años sin tratamiento radiante previo. Excluyendo los estudios en menores de 18 años, metaanálisis, revisiones y tratamientos de reirradiación.

\section{RESULTADOS}

Un total de 77 publicaciones fueron encontradas. En el filtrado fueron eliminados los artículos que se repetían, quedando treinta y uno. Cinco publicaciones fueron excluidas, por diversas causas: el idioma, no tener acceso al texto completo, no reportar los resultados clínicos por la intervención y uno por ser un protocolo sin tener aún publicados los resultados. Un total de 26 artículos se eligieron para el análisis cualitativo (Figura 1).

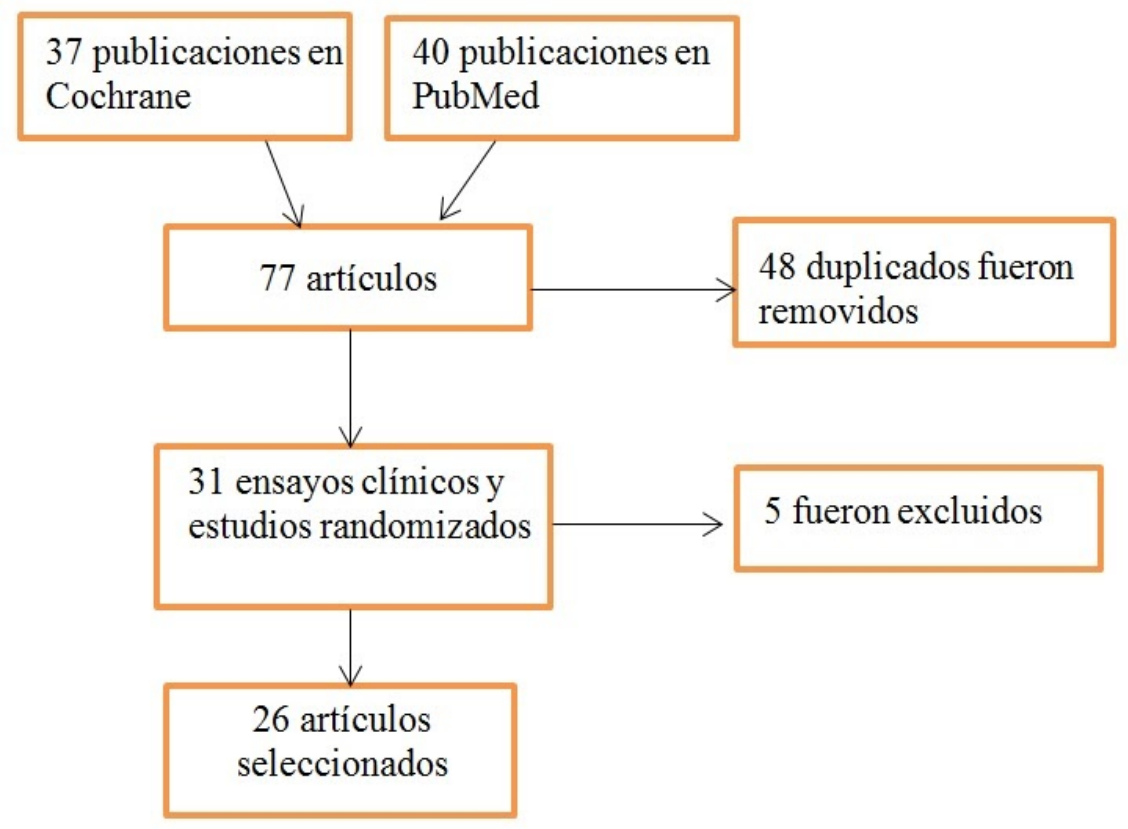

Figura 1. Flujograma de los artículos incluidos y excluidos en la revisión

La mayoría de las series son estudios restrospectivos de cohortes pequeñas de centro único. La SRS tiene buenos resultados disminuyendo el riesgo de hemorragia posterior al tratamiento, sin determinar un deterioro significativo de la neurocognición o no mayor al impacto que genera la alteración de la hemodinámica cerebral. La expectativa de vida puede ser un parámetro importante, la balanza se inclina hacia la SRS en pacientes con edad avanzada cuando se compara con microcirugía $(10,11)$.

La SRS tiene un papel preponderante en MAVs profundas o situadas en área elocuente, grado III o mayor de SM, donde la morbilidad quirúrgica puede ser más importante. Reportan tasa de obliteración de las MAVs de un $61 \%$ a $80 \%$ (12-17). Vijayakumar y colaboradores (18), reportaron una tasa de $46,5 \%$ en su estudio, pero el seguimiento fue corto, solo 15 pacientes de los 37 dentro del estudio, tuvieron un seguimiento de más de 36 meses. Las principales variables asociadas a una mejor respuesta son el menor tamaño de la MAV, no embolización previa y la dosis prescrita, siendo la probabilidad de curación de la MAV aproximadamente de 70, 80 y $90 \%$ para dosis de irradiación de 16, 18, y 20 Gy respectivamente $(14,16,19,20)$. Ding y colaboradores (14), encontraron mejor respuesta en pacientes con nido $<4 \mathrm{~cm}^{3}$ tratados con dosis $>18$ Gy. En otro estudio del mismo autor dosis de al menos 22 Gy se asoció a una tasa significativamente 
mayor de resultado óptimo, definido como la obliteración de la MAV, sin hemorragia pos SRS y sin complicaciones radioinducidas permanentes y sintomáticas (15).

La obliteración luego de la SRS no ocurre inmediatamente, existe un periodo de latencia de dos a tres años antes de la obliteración total del nido, tiempo en el cual los pacientes siguen en riesgo de hemorragia. El riesgo de sangrado no aumenta en el período de latencia entre la SRS y la obliteración, con respecto a la historia natural de las MAVs. Hay reportes de tasa de hemorragia anual posterior a la SRS para una MAV no rota de 0.9 - 1.9\% (14-16, 21). Comparando el riesgo de hemorragia antes (historia natural) y después de la SRS (periodo de latencia), el riesgo disminuye luego de SRS. El beneficio fue similar en el análisis cuando se compara MAV con o sin hemorragia previa $(21,22)$. La SRS de fracción única parece ser más adecuada para volúmenes pequeños a moderados $\left(10 \mathrm{a} 12 \mathrm{~cm}^{3}\right.$, diámetro de $3 \mathrm{~cm}$ aproximadamente) en MAVs asintomáticas o con alto riesgo de sangrado. El promedio de dosis usada en los estudios más recientes es de $20 \mathrm{~Gy}$.

Wang y colaboradores (23), en 116 pacientes tratados con Acelerador Lineal, reportaron una tasa de obliteración del $82 \%$ y una morbilidad menor al $5 \%$. Durante el período de latencia después de SRS, los cambios sintomáticos atribuibles a efectos adversos de la radiación ocurren aproximadamente en el 10\% de los pacientes, este riesgo varía según la ubicación de MAV, el volumen blanco y la dosis al tejido normal circundante. Cambios neurológicos permanentes debido a radionecrosis se pueden observar hasta en el $2 \%$ al $3 \%$ de los pacientes $(24,25)$. La incidencia de neoplasias radioinducidas luego de SRS es un fenómeno extremadamente raro, del orden del $0,64 \%$ (26).

Luego de muchos estudios con resultados prometedores del uso de intervencionismo en MAVs, el polémico estudio ARUBA (A Randomised trial of Unrupted Arteriovenous malformations) (27), un ensayo prospectivo multicentrico, cuestionó el beneficio en MAV no rotas. Incluyó pacientes mayores de 18 años, asignados a tratamiento médico con terapia intervencionista (neurocirugía, embolización o SRS) o tratamiento médico exclusivo. El objetivo primario fue establecer el tiempo transcurrido desde el diagnóstico hasta un accidente cerebrovascular sintomático o muerte. Presentó un cierre anticipado debido a la superioridad del grupo de manejo médico. Con 33 meses de seguimiento el riesgo de muerte o accidente cerebrovascular fue menor en el grupo de tratamiento médico con significancia estadística. Estos resultados persisten en su último reporte con cinco años de seguimiento. Las principales limitaciones al ensayo ARUBA son el número reducido de pacientes que incluyó, el tiempo corto de estudio (tres años) y la heterogeneidad en el brazo de tratamiento intervencionista. Al incluir diversas modalidades de tratamiento, formó un grupo heterogéneo que requiere resultados según cada intervención con las tasas de obliteración obtenidas, que por resultados de otros estudios son diferentes, se estima una mayor tasa de obliteración con la microcirugía, además el tratamiento multimodal podría tener mayor morbilidad. Lo más llamativo fue la elevada tasa de hemorragia y muerte que reportó en el grupo intervencionista, mucho mayor que otros estudios previos.

Esto motivó más investigaciones al respecto. Se realizaron varios estudios con pacientes seleccionados según los criterios de selección que el brazo intervencionista del ARUBA, para así comparar sus resultados. Kim y colaboradores (13), en un estudio retrospectivo con un seguimiento medio de 4,6 años reportó menor tasa de hemorragia y muerte en pacientes tratados con radiocirugía. Ding y colaboradores reportaron una tasa de hemorragia anual posterior a SRS de $0.9 \%$, cambios radio inducidos sintomáticos $\mathrm{y}$ permanentes en $11.2 \%$ y $2.6 \%$ de los pacientes, respectivamente. Mortalidad posterior al tratamiento de $4.3 \%$ (14). Llevando a cabo incluso un análisis según el grado de SM persiste el beneficio de la SRS, con una menor tasa de mortalidad y hemorragia $(15,28)$. Otros estudios que incluyeron las diferentes modalidades de tratamiento, incluso multimodal, con criterios de selección iguales a ARUBA, 
encontraron tasa de muerte o hemorragia sintomática del 7-8\% (29, 30). Estos datos contradicen los hallazgos de la ARUBA y sugieren que el tratamiento de las MAV cerebrales no rotas es seguro y eficaz, pero a su vez tienen las limitaciones de un estudio retrospectivo.

\section{Tratamiento multimodal}

Para las MAVs más pequeñas, varios estudios han sugerido que la embolización previa es un factor predictivo negativo de la obliteración por SRS (14, 31). Sin embargo, la reducción del tamaño por embolización aumentó la tasa de obliteración en las grandes MAV según lo informado por Veznedaroglu y colaboradores (32). Tratar MAVs gigantes con SRS o cirugía resulta en mayor morbilidad y mortalidad que las malformaciones de menor tamaño. Las indicaciones aceptadas para el tratamiento de estas lesiones incluyen hemorragia repetida, déficits neurológicos progresivos, convulsiones intratables y otros síntomas graves. En estos pacientes el tratamiento multimodal tiene mejores resultados y la SRS tiene beneficio antes de una microcirugía, al disminuir el tamaño del nido. Regímenes fraccionados de 7 Gy en cuatro sesiones, y 6-6.5 Gy en cinco fracciones pueden traer resultados aceptables entre las tasas de obliteración y las de posibles complicaciones asociadas al tratamiento. Los resultados de series que utilizan tales enfoques muestran tasas de obliteración de $35 \%$ a 58\% y tasas de morbilidad neurológica permanente relacionada con el tratamiento del $2 \%$ al $15 \%$ (33). Finalmente, se puede considerar una estrategia de SRS por etapas, esto conlleva dividir la MAV en dos o más sub-volúmenes, tratando cada una de estas secciones en sesiones separadas con intervalos de 2 a 9 meses $(34,35)$.

La SRS ha demostrado eficacia en MAVs gigantes donde las opciones terapéuticas son limitadas, pues en ellas la cirugía es difícil, con alta morbimortalidad, y la embolización muy problemática. En particular, existe debate entre hacer un tratamiento único o multimodal a las MAVs de alto riesgo, pues un tratamiento parcial podría aumentar la tasa de hemorragia hasta un 10,4\%.
La microcirugía en estos casos podría dejarse para pacientes con hemorragia previa, con déficit neurológico debido a compresión por la MAV, por robo vascular, o la existencia de aneurisma arterial o intranidal asociado.(10)

\section{CONCLUSIONES}

La SRS de fracción única es un método seguro, eficaz, y con baja morbilidad para el tratamiento de MAVs menores a $3 \mathrm{~cm}$, con especial ventaja para MAVs profundas o ubicadas en área cerebral elocuente. Para MAVs grandes, la SRS fraccionada puede ser utilizada como monoterapia o en un enfoque multimodal, ya sea adyuvante a la embolización o neoadyuvante a la microcirugía. Las MAVs son lesiones complejas, donde el balance entre el beneficio de la intervención y el riesgo de secuelas debe ser valorado de forma meticulosa. El enfoque multidisciplinario es de crucial importancia para la elección del plan terapéutico que más se ajuste a cada caso en particular.

\section{CONTRIBUCIONES DE LOS AUTORES:} Concepcion y diseño del articulo: LR, SA. Recolección, análisis e interpretación de datos: LR, MR. Redacción: LR, MR. Revision critica y aprobación final: KS. Responsable de la veracidad: LR, MR, SA, KS.

CONFLICTOS DE INTERESES: Declaramos que no existen potenciales conflictos de intereses con esta publicación.

\section{REFERENCIAS}

1.Vlaskou Badra E, Ermiş E, Mordasini P, Herrmann E. Radiosurgery and radiotherapy for arteriovenous malformations: outcome predictors and review of the literature. J neurosurgical sci. 2018; 62(4): 490-504. DOI: $\underline{10.23736 / \mathrm{S} 0390-5616.18 .04406-5}$

2. Brown, R. D., Jr, Wiebers, D. O., Forbes, G., O'Fallon, W. M., Piepgras, D. G., Marsh, W. R., \& Maciunas, R. J. The natural history of unruptured intracranial arteriovenous malformations. J Neurosurg. 1988; 68(3): 352-357. 
3. Van Beijnum J, Lovelock CE, Cordonnier C, Rothwell PM, Klijn CJ, Salman RA. Outcome after spontaneous and arteriovenous malformation-related intracerebral haemorrhage: population-based studies. Brain. 2009; 132( $\mathrm{Pt}$ 2): $537-543$.

https:/doi.org/10.1093/brain/awn318

4. Laakso, A., Dashti, R., Seppänen, J., Juvela, S., Väärt, K., Niemelä, M., et.al. Long-term excess mortality in 623 patients with brain arteriovenous malformations. Neurosurgery. $\quad 2008 ; \quad 63(2)$ : 244-53. https:/doi.org/10.1227/01.NEU.0000320439.27895.24

5. Hernesniemi JA, Dashti R, Juvela S, Vaart K, Niemela M, Laakso A. Natural history of brain arteriovenous malformations: a long-term follow-up study of risk of hemorrhage in 238 patients. Neurosurgery. 2008; 63(5): $823-831$. https:/doi.org/10.1227/01.NEU.0000330401.82582.5 E

6. Stapf, C., Mast, H., Sciacca, R. R., Choi, J. H., Khaw, A. V., Connolly, E. S., et.al. Predictors of hemorrhage in patients with untreated brain arteriovenous malformation. Neurology. 2006; 66(9): 1350-5. https:/doi.org/10.1212/01.wnl.0000210524.68507.87

7. Stapf C, Mohr JP, Pile-Spellman J, Sciacca RR, Hartmann A, Schumacher HC, et al. Concurrent arterial aneurysms in brain arteriovenous malformations with haemorrhagic presentation. J Neurol Neurosurg Psychiatry. 2002; 73(3), 294-298. https://doi.org/10.1136/jnnp.73.3.294

8. Pollock BE, Lunsford LD. A call to define stereotactic radiosurgery. Neurosurgery. 2004; 55: 1371-1373. https://doi.org/10.1227/01.neu.0000143613.13759.d4

9. Sallabanda K, Ley Urzaiz L, Lorenzana Honrado L. Radiocirugía en las Malformaciones Arterio Venosas. En: Samblas J, Sallabanda K, Martínez R, Calvo F. Radiocirugía: fundamentos, avances tecnológicos, indicaciones y resultados. Madrid: Aran; 2012. p. 319334.

10. Guo, W. Y., Lee, S. M., Chang, Y. C., \& Pan, H. C. The impact of arteriovenous malformation radiosurgery on the brain: From morphology and perfusion to neurocognition. Stereotactic and functional neurosurgery. 2006; 84(4), 162-169. https://doi.org/10.1159/000094955
11. Nataf F, Schlienger M, Bayram M, Ghossoub M, George B, Roux FX. Microsurgery or radiosurgery for cerebral arteriovenous malformations? A study of two paired series. Neurosurgery. 2007; 61(1): 39-50. https://doi.org/10.1227/01.neu.0000279722.60155.d3

12. Yen, C. P., Ding, D., Cheng, C. H., Starke, R. M., Shaffrey, M., \& Sheehan, J. Gamma Knife surgery for incidental cerebral arteriovenous malformations. Journal of neurosurgery. 2014; 121(5), 1015-1021. https://doi.org/10.3171/2014.7.JNS131397

13. Kim, B. S., Yeon, J. Y., Kim, J. S., Hong, S. C., Shin, H. J., \& Lee, J. I. Gamma Knife Radiosurgery for ARUBA-Eligible Patients with Unruptured Brain Arteriovenous Malformations. Journal of Korean medical science. 2019; 34(36), e232. https://doi.org/10.3346/jkms.2019.34.e232

14. Ding, D., Yen, C. P., Xu, Z., Starke, R. M., \& Sheehan, J. P. Radiosurgery for patients with unruptured intracranial arteriovenous malformations. $\underline{\mathbf{J}}$ Neurosurgery. $\quad 2013 ; \quad 118(5)$ : 958-966. https://doi.org/10.3171/2013.2.JNS121239

15. Ding, D., Starke, R. M., Kano, H., Mathieu, D., Huang, P., Kondziolka, et.al. Radiosurgery for Cerebral Arteriovenous Malformations in A Randomized Trial of Unruptured Brain Arteriovenous Malformations (ARUBA)-Eligible Patients: A Multicenter Study. Stroke. 2016; 47(2), 342-349. https://doi.org/10.1161/STROKEAHA.115.011400

16. Han, J. H., Kim, D. G., Chung, H. T., Park, C. K., Paek, S. H., Kim, J. E., et. al. Clinical and neuroimaging outcome of cerebral arteriovenous malformations after Gamma Knife surgery: analysis of the radiation injury rate depending on the arteriovenous malformation volume. Journal of neurosurgery. 2008; 109(2), 191-198. https://doi.org/10.3171/JNS/2008/109/8/0191

17. Hanakita, S., Shin, M., Koga, T., Igaki, H., \& Saito, N. Risk Reduction of Cerebral Stroke After Stereotactic Radiosurgery for Small Unruptured Brain Arteriovenous Malformations. Stroke. 2016; 47(5), 1247-1252.

https://doi.org/10.1161/STROKEAHA.116.013132 
18. Javalkar V, Pillai P, Vannemreddy P, Caldito G, Ampil F, Nanda A. Gamma knife radiosurgery for arteriovenous malformations located in eloquent regions of the brain. Neurol India. 2009; 57: 617-21. https://www.neurologyindia.com/text.asp?2009/57/5/ $\underline{617 / 57818}$

19. Flickinger JC, Pollock BE, Kondziolka D, Lunsford LD. A dose-response analysis of arteriovenous malformation obliteration after radiosurgery. Int $\mathrm{J}$ Radiat Oncol Biol Phys. 1996; 36(4): 873-879. https://doi.org/10.1016/s0360-3016(96)00316-1

20. Donald A. Ross, Howard M. Sandler, James M. Balter, James A. Hayman, John Deveikis, Donna L. Auer. Stereotactic Radiosurgery of Cerebral Arteriovenous Malformations with a Multileaf Collimator and a Single Isocenter, Neurosurgery. 2000; 47(1): 123130. https://doi.org/10.1097/00006123-200007000$\underline{00026}$

21. Yen, C. P., Sheehan, J. P., Schwyzer, L., \& Schlesinger, D. Hemorrhage risk of cerebral arteriovenous malformations before and during the latency period after GAMMA knife radiosurgery. Stroke. 2011; 42(6), 1691-1696. https://doi.org/10.1161/STROKEAHA.110.602706

22. Maruyama, K., Kawahara, N., Shin, M., Tago, M., Kishimoto, J., Kurita, H., et.al. The risk of hemorrhage after radiosurgery for cerebral arteriovenous malformations. The New England journal of medicine. 2005; 352(2), 146-153. https://doi.org/10.1056/NEJMoa040907

23. Wang YC, Huang YC, Chen HC, Wei KC, Chang $\mathrm{CN}$, Lee ST, et. al. Linear accelerator stereotactic radiosurgery in the management of intracranial arteriovenous malformations: long-term outcome. Cerebrovasc Dis. 2014; 37(5): 342-349. https://doi.org/10.1159/000360756

24. Steiner L, Lindquist C, Adler JR, Torner JC, Alves $\mathrm{W}$, Steiner M. Clinical outcome of radiosurgery for cerebral arteriovenous malformations. J Neurosurg. 1992; 77: 1-8. Disponible en: https://doi.org/10.3171/jns.1992.77.1.0001

25. Parkhutik, V., Lago, A., Aparici, F., Vazquez, J. F., Tembl, J. I., Guillen, L., et.al. Late clinical and radiological complications of stereotactical radiosurgery of arteriovenous malformations of the brain. Neuroradiology 2013; 55(4), 405-412. https://doi.org/10.1007/s00234-012-1115-8

26. Starke, Robert \& Po Yen, Chun \& Chen, Ching-Jen \& Ding, Dale \& Mohila, Carrie \& Jensen, Mary \& Kassell, Neal \& P Sheehan, Jason. An Updated Assessment of the Risk of RadiationInducedNeoplasia After Radiosurgery of Arteriovenous Malformations. World Neurosurgery. 2014; 395-401. https://doi.org/10.1016/j.wneu.2013.02.008

27. Mohr JP, Parides MK, Stapf C, Moquete E, Moy CS, Overbey JR, et al. Medical management with or without interventional therapy for unruptured brain arteriovenous malformations (ARUBA): a multicentre, non-blinded, randomised trial. Lancet. 2014; Lancet. 2014 Feb 15;383(9917):614-21. Epub 2013 Nov 20. Disponible en: https://doi.org/10.1016/S0140$\underline{6736(13) 62302-8}$

28. Ding, D., Starke, R. M., Kano, H., Lee, J. Y., Mathieu, D., Pierce, J., et. al. Stereotactic radiosurgery for Spetzler-Martin Grade III arteriovenous malformations: an international multicenter study. Journal of neurosurgery. 2017; 126(3), 859871.: https://doi.org/10.3171/2016.1.JNS152564

29. Link, T. W., Winston, G., Schwarz, J. T., Lin, N., Patsalides, A., Gobin, P., et.al. Treatment of Unruptured Brain Arteriovenous Malformations: A Single-Center Experience of 86 Patients and a Critique of the A Randomized Trial of Unruptured Brain Arteriovenous Malformations (ARUBA) Trial. World neurosurgery. $\quad 2018 ; 120, \quad$ e1156-e1162. https://doi.org/10.1016/j.wneu.2018.09.025

30. Lang, M., Moore, N. Z., Rasmussen, P. A., \& Bain, M. D. Treatment Outcomes of A Randomized Trial of Unruptured Brain Arteriovenous MalformationEligible Unruptured Brain Arteriovenous Malformation Patients. Neurosurgery. 2018; 83(3), 548-555. https://doi.org/10.1093/neuros/nyx506

31. Raboud, M., Tuleasca, C., Maeder, P., Schiappacasse, L., Marguet, M., Daniel, R. T., \& Levivier, M. Gamma knife radiosurgery for arteriovenous malformations: general principles and preliminary results in a Swiss 
cohort. Swiss medical weekly. 2018; 148, w14602. https://doi.org/10.4414/smw.2018.14602

32. Veznedaroglu E, Andrews DW, Benitez RP, Downes $\mathrm{MB}$, Werner-Wasik M, Rosenstock $\mathrm{J}$, et al. Fractionated stereotactic radiotherapy for the treatment of large arteriovenous malformations with or without previous partial embolization. Neurosurgery. 2008 Feb; $62 \quad$ Suppl 2: 763-75. https://doi.org/10.1227/01.neu.0000316280.99500.99

33. Chang SD, Marcellus ML, Marks MP, Levy RP, Do HM, Steinberg GK. Multimodality treatment of giant intracranial arteriovenous malformations. Neurosurgery. $\quad 2003 ; \quad 53$ : $1-11$. https://doi.org/10.1227/01.neu.0000068700.68238.84
34. Chung WY, Shiau CY, Wu HM, Liu KD, Guo WY, Wang LW, Pan DH. Staged radiosurgery for extralarge cerebral arteriovenous malformations: method, implementation, and results. J Neurosurg. 2008; 109 (sup$\mathrm{pl})$ : 65-72. https://doi.org/10.3171/JNS/2008/109/12/S11

35. Unkelbach, J., Bussière, M. R., Chapman, P. H., Loeffler, J. S., \& Shih, H. A. Spatiotemporal Fractionation Schemes for Irradiating Large Cerebral Arteriovenous Malformations. International journal of radiation oncology, biology, physics. 2016; 95(3): 1067-1074.

https://doi.org/10.1016/j.ijrobp.2016.02.001 